\title{
Unexpected delayed death after manual strangulation: need for careful examination in the emergency room
}

\author{
M. Di Paolo1, B. Guidi1, L. Bruschini2, G. Vessio³, \\ R. Domenici'1, N. Ambrosino 4
}

\begin{abstract}
Unexpected delayed death after manual strangulation: need for careful examination in the emergency room. M. Di Paolo, B. Guidi, L. Bruschini, G. Vessio, R. Domenici, N. Ambrosino.

The aim of this case report was to demonstrate the importance of detailed clinical assessment in victims of attempted manual strangulation, to prevent the occurrence of delayed death due to an airway collapse; and to provide an update on clinical management of these patients. An elderly male patient presented with a sore throat and speech impairment after attempting manual strangulation. Physical examination showed reddish skin of the neck, an ex-
\end{abstract}

tensive haematoma of the hard palate and anterior tongue. Flexible laryngoscopy failed to show any swelling of the hypopharynx or larynx. A few hours after presentation, the patient developed acute dyspnoea and died. Autopsy and post-mortem CT scan showed a haematoma in the thyro-epiglottal space. In conclusion, victims of manual strangulation can survive despite internal neck injury which can lead to delayed fatal airway collapse. This is because often there are few or no signs of assault, therefore medical evaluation should be thorough and timely. Sometimes immediate tracheotomy can be life saving.

Monaldi Arch Chest Dis 2009; 71: 3, 132-134.

Keywords: Manual strangulation, Delayed death, Autopsy.

1 Institute of Legal Medicine, University of Pisa,

2 ENT Unit, University of Pisa,

3 ENT Unit, University of Siena,

4 Pulmonary Unit, Cardio-Thoracic Department, University Hospital, Pisa, Italy.

Correspondence: Marco Di Paolo, MD, Institute of Legal Medicine, Via Roma 55, 56100 Pisa, Italy; e-mail: marcodipaolo@med.unipi.it

\section{Introduction}

Manual strangulation - or throttling - is a form of blunt neck trauma. The victim suffers fatal pressure on the airways, blood vessels and nervous structure of the neck, which leads to anoxia, irreversible brain damage and rapid death. Some victims of strangulation survive [1] and several cases of delayed death after strangulation have been reported in literature [2-4]. The neck injury may not be recognised in the emergency room due to lack of visible external signs or mucosal damage. If patients are relatively asymptomatic, the extent of soft tissue, cartilage and neurovascular damage may be underestimated during physical examination, especially in the absence of respiratory distress. Fatal airway collapse may occur more than 24 hours after assault [5].

\section{Case report}

An 80-year-old man presented at the emergency unit four hours after being seized by the neck by his 65 -year-old wife. The patient complained of a sore throat and speech impairment. Medical history included Parkinson syndrome and COPD; medication included salicylates. On initial physical examination vital signs were BP 150/55, respiration 20, oxygen saturation $92 \%$ on room air. Physical examination showed reddening of the skin of the neck and anterior neck swelling. The neck was diffusely sore without subcutaneous emphysema. Examination of the oral cavity showed a haematoma of the hard palate and anterior tongue. The soft palate and oropharynx were diffusely edematous. Flexible laryngoscopy failed to show any swelling of the hypopharynx or larynx. About 3 hours after presentation, patient developed acute dyspnea. Emergency tracheotomy was performed, but the patient died in a few minutes.

External post-mortem examination showed an oval ecchymosis measuring $3.4 \times 3 \mathrm{~cm}$ (fingertip bruise) in the right submandibular space, severe intradermal bruising around the neck, mainly visible on the left side. There was a slight abrasion under the left angle of the jaw and lateral cervical swelling. Petechial haemorrhaging of the conjunctiva and facial skin was absent. Autopsy showed petechiae on the surface of the heart and lungs. Fluidity of blood in the heart chambers and great vessels was persistent. Lung congestion and oedema were also observed. A separate layer-by-layer dissection of the neck revealed bulky subcutaneous haemorrhagic flow in the left lateral cervical 


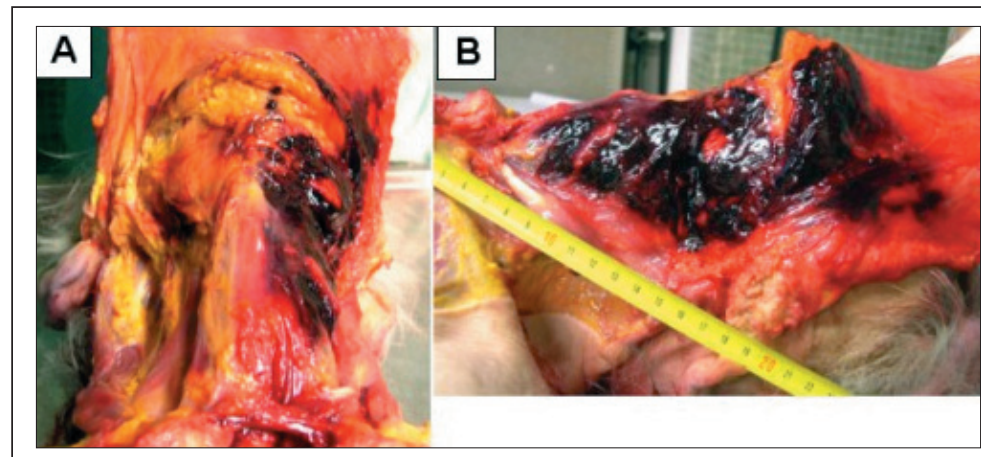

Fig. 1. - Frontal (A) and lateral (B) view of subcutaneous plane of neck showing bulky subcutaneous haemorrhagic flow in left lateral cervical region and diffuse interstitial haemorrhagic infiltration.

region (fig. 1) and diffuse interstitial haemorrhagic infiltration, with perifascial muscle involvement but no internal lesions of the strap muscles.

Post-mortem radiological evaluation, by spiral CT scan of neck organs, showed a bulky haematoma, which displaced the base of the tongue and obstructed the upper airways, in the thyro-epiglottal space (fig. 2). Anatomical examination (transverse longitudinal section) confirmed the haematoma in the thyro-epiglottal space and involvement of the base of the tongue, together with haemorrhagic infarction of the false vocal cords and submandibular and submaxillary glands (fig. 3). The laryngeal cartilage and hyoid bone were intact.

\section{Discussion}

Manual strangulation causes asphyxia through compression of the neck. Death may occur by a combination of respiratory, circulatory and neurological factors. Survival after throttling is possible. Due to the static nature of the compressive forces involved in strangulation, victims may present at casualty with deceptively minor signs and symptoms. There may be few, if any, clear external signs of injury to the neck skin [1]. McClane et al

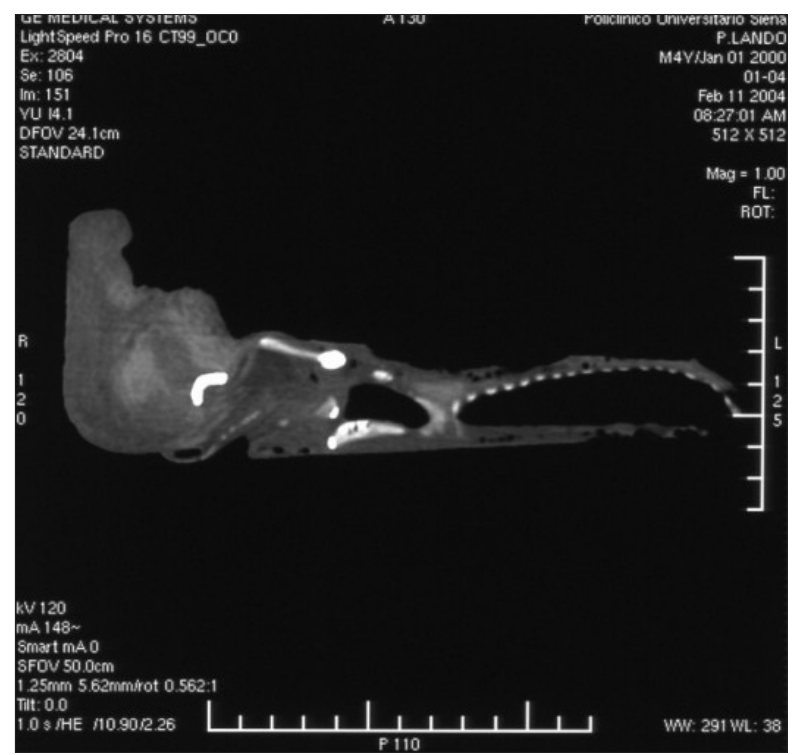

Fig. 2. - Spiral CT scan showing bulky haematoma, displacing base of tongue and obstructing upper airways, in thyro-epiglottal space.
[6] report that $50 \%$ of victims surviving strangulation had no visible neck signs and $35 \%$ had minor injuries.

Damage to the cartilaginous framework without manifest mucosal lacerations or submucosal haemorrhage is described in literature $[7,8]$. The upper airway may also appear normal under intact mucosa, in the presence of hyoid bone fractures [5]. Delayed oedema, engorgement, haematoma, dislocated laryngeal fractures and vocal cord immobility may all contribute to airway failure after garrotting [5].

When the laryngeal cartilaginous framework is intact, soft tissue oedema and haematoma of the supraglottis are the most frequent causes of upper airway distress [9]. Swelling may develop quickly or insidiously, leading to upper airway occlusion several hours after assault [10]. Visible neck signs are rarely present immediately after aggression [11]. These features explain why victims of strangulation are often clinically underestimated.

Due to the fact that damage caused by manual strangulation can be fatal even when there are few or no signs of assault, medical evaluation should be thorough and timely. Assessment of internal injury is not a lengthy procedure and can save lives.

For as long as 36 hours after assault, the victim can develop oedema of the supraglottal and oropharyngeal soft tissue, causing airway obstruction [1].

Variables include the mode and force of compression as well as patient age and general medical condition. Though it is difficult to recognise which patients could be susceptible haematoma formation, vigilance is necessary in all cases [14].

The treatment of victims of attempted strangulation depends on signs and symptoms. Since the main aim is to protect the airways, patients with mild symptoms should be investigated in the same detail as patients with more significant signs, such as visible neck injuries, dyspnea and neurological signs [11]. Chest $X$ ray and ultrasonography are necessary to detect fractures of larynx or hyoid bone and tracheal deviation caused by oedema or haematoma. CT scan and/or MRI of neck structures are recommended for a more exhaustive assessment. Carotid Doppler ultrasound may be appropriate in case of neurological lateralisation.

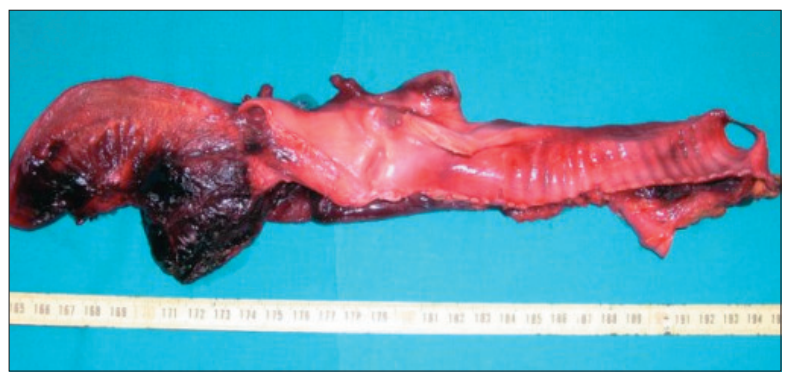

Fig. 3. - Transverse longitudinal section of tongue, larynx and trachea showing haematoma in thyro-epiglottal space and involvement of base of tongue. 
Victims of attempted strangulation should be admitted to hospital and kept under observation, monitoring vital signs, respiratory function and neurological status. Particular care is required for patients under the influence of drugs (such as anticoagulants) or alcohol [11, 12].

In conclusion, extensive injury to the laryngotracheal region may have few outward signs after blunt trauma to the neck. Immediate, aggressive management of the airways is essential for successful outcome.

In similar circumstances, the lack of an adequate diagnostic procedure undoubtedly leads to medical malpractice hypotesis.

\section{References}

1. Funk M, Schuppel J. Strangulation injuries. Wisconsin Medical Journal 2003; 102: 41-45.

2. Anscombe AM, Knight BH. Delayed death after pressure on the neck: possible causal mechanism and implications for mode of death in manual strangulation discussed. Forensic Sci Int 1996; 78: 193-197.

3. Knight B. Fatal pressure on the neck. In B. Knight, Editor: Forensic Pathology (2nd ed.), Arnold, London, 1996, 361-391.
4. Brinkmann B, Koops E, Wischlusen F, Kleiber M. Compression of the neck and arterial obstruction. $Z$. Rechtsmed 1981; 7: 59-73.

5. Kuriloff DB, Pincus RL. Delayed airway obstruction and neck abscess following manual strangulation injury. Ann Otol Rhinol Laryngol 1989; 98: 824-827.

6. McClane GE, Styrack GB, Hawley D. A review of 300 attempted strangulation cases, part II: Clinical evaluation of the surviving victim. $J$ Emerg Med 2001; 21: 311-315.

7. Peppard SB. Transient vocal paralysis following strangulation injury. Laryngoscope 1982; 92: 31-93.

8. Vanezis P, Pathology of Neck Injury. Butterworths, London, 1989.

9. Schaffer SD. The treatment of acute external laryngeal injuries "state of the art". Arch Otolaryngol Head Neck Surg 1991; 117: 35-39.

10. Keogh IJ, Rowley H, Russel J. Critical airway compromise caused by neck haematoma. Clin Otolaryngol 2002; 27: 244-245.

11. Clarot F, Vaz E, Papin F, Proust B. Fatal and non-fatal bilateral delayed carotid artery dissection after manual strangulation. Forensic Sci Int 2005; 149: 143150.

12. Boster SR, Bergin JJ. Upper airway obstruction complication warfarin therapy: with a note on reversal of warfarin toxicity. Annal Emerg Med 1983; 12: 711715 .

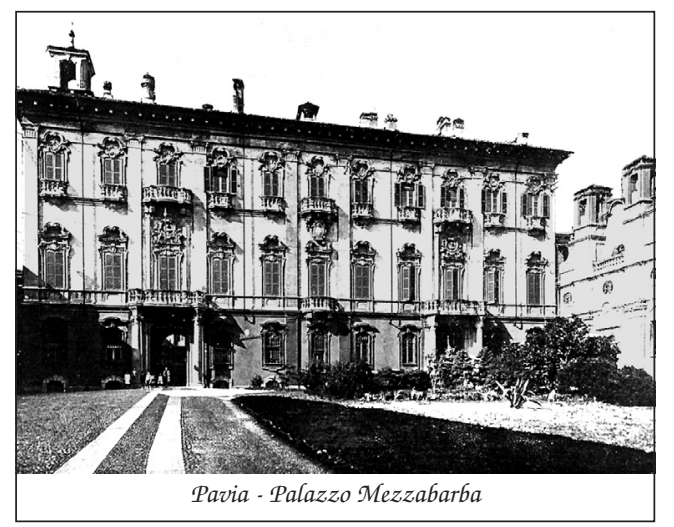

